\title{
MoleCUlaR DISSECtion OF hOSt CELL INVASION bY the ApICOMPLEXANS: THE GLIDEOSOME
}

SOLDATI-FAVRE D.*

\section{Summary:}

Gliding motility is an essential and fascinating apicomplexantypical adaptation to an intracellular lifestyle. Apicomplexan parasites rely on gliding motility for their migration across biological barriers and for host cell invasion and egress. This unusual substrate-dependent mode of locomotion involves the concerted action of secretory adhesins, a myosin motor, factors regulating actin dynamics and proteases. During invasion, complexes of soluble and transmembrane micronemes proteins (MICs) and rhoptry neck proteins (RONs) are discharged to the apical pole of the parasite, some protein acts as adhesins and bind to host cell receptors whereas others are involved in the moving junction formation. These complexes redistribute towards the posterior pole of the parasite via a physical connection to the parasite actomyosin system and are eventually released from the parasite surface by the action of parasite proteases.

KEY WORDS : Toxoplasma gondii, Plasmodium falciparum, Apicomplexa, adhesin, microneme, actin, myosin, proteases.

] ntracellular pathogens have evolved a great variety of smart strategies to establish infection, evading the host cellular and immune defences and adapting exquisitely to their intracellular niches. While bacterial pathogens can easily enter non-professional phagocytes by induced-phagocytosis, such a task is more challenging for the significantly larger protozoan parasites. Apicomplexans actively enter their host cells relying on an unusual form of motility, which requires contact with the substrate and does not depend on cilia or flagella (Sibley, 2004). Gliding is crucial for the establishment of infection by most apicomplexan parasites contributing to migration across biological barriers and host cell invasion and egress. This active mode of penetration presents numerous advantages for these pathogens:

ABBREVIATIONS

PRF, profilin; FRM, formin; FH2, forming homology domain 2; ADF, actin depolymerizing factor; ARP, actin related protein; MIC, microneme protein; RON, rhoptry neck protein; MHC, myosin heavy chain; MLC, myosin light chain; ROM, rhomboid like proteases; MPP, micorneme protein protease; IMC, inner membrane complex; MAR microneme adhesive repeat.

\footnotetext{
* Department of Microbiology and Molecular Medicine, CMU, University of Geneva, 1, rue Michel-Servet, 1211 Geneva 4, Switzerland. Tel.: + 41223795672 - Fax: + 41223795702.

E-mail: dominique.soldati-favre@medecine.unige.ch
}

i) the parasite can enter almost any cell types including red blood cells as in the case of Plasmodium species; ii) this smooth mode of entry does not stimulate the host cell defences mechanisms; iii) this process leads to the formation of a unique parasitophorous vacuole, a non-phagosomal, membrane bound compartment that is segregated from most cellular trafficking pathways enabling the parasite to survive even in macrophages and for a prolonged time (Joiner et al., 1990). Host cell entry by the apicomplexans is thought to follow a broadly similar mechanism involving multiple steps. After contact with the host cell, the parasite reorientates such that its apical pole points towards and interacts with the host cell plasma membrane. Microneme secretion is triggered, as a result of a calciummediated signal, and proteins are discharged on the parasite surface. Some of these microneme proteins are thought to mediate high affinity, specific interactions with host cell receptors. A "tight junction" is formed between the parasite and host, which translocates towards the rear of the parasite via interactions between the microneme protein cytoplasmic tails and a cortical parasite actin-myosin system. This process provides the traction for invasion. Aldolase, an F-actin binding protein has been identified as a protein capable of connecting the host receptors-parasite ligands complexes to the parasite actomyosin system. Indeed studies both in T. gondii and Plasmodium species showed that the tails of TgMIC2 (Jewett \& Sibley, 2003) PbTRAP (Buscaglia et al., 2003) and TRAP family members (Baum et al., 2006b) bind to aldolase. A recent study demonstrated that the acidic domain of WASP binds very efficiently to aldolase but definitive experimental evidence demonstrating that aldolase is physiologically involved in actin nucleation either in mammalian cells or in apicomplexans is still lacking (Buscaglia et al., 2006).

Immediately following microneme discharge the rhoptry organelles secrete their contents. Some rhoptry proteins (along with the microneme protein AMA1) have recently been demonstrated to contribute to the formation of the moving junction, although their precise role is unknown (Alexander et al., 2005; Carruthers \& Boothroyd, 2007; Dubremetz, 2007; Lebrun et al., 
2005). Other rhoptry materials and proteins are involved in the genesis of the parasitophorous vacuole and also act as effector molecules remodelling host cells functions (Boothroyd \& Dubremetz, 2008; Bradley \& Sibley, 2007). Migration across barriers involves the gliding machinery but the rhoptries are most likely not mobilised and thus a parasitophorous vacuole is not formed. Invasion and egress share common mechanistic features and factors, and in some respects invasion can be thought to begin at the moment of egress, as from this point on the parasite is primed and ready for invasion (Hoff \& Carruthers, 2002). Toxoplasma is a well-developed genetic system (Roos et al., 1994) that has been extensively exploited to dissect the mechanism of gliding and host cell invasion at the molecular level. The major findings regarding the gliding machinery or the "Glideosome" appear to hold true for other members of the phylum Apicomplexa (Fig. 1) (Keeley \& Soldati, 2004; Soldati et al., 2004).
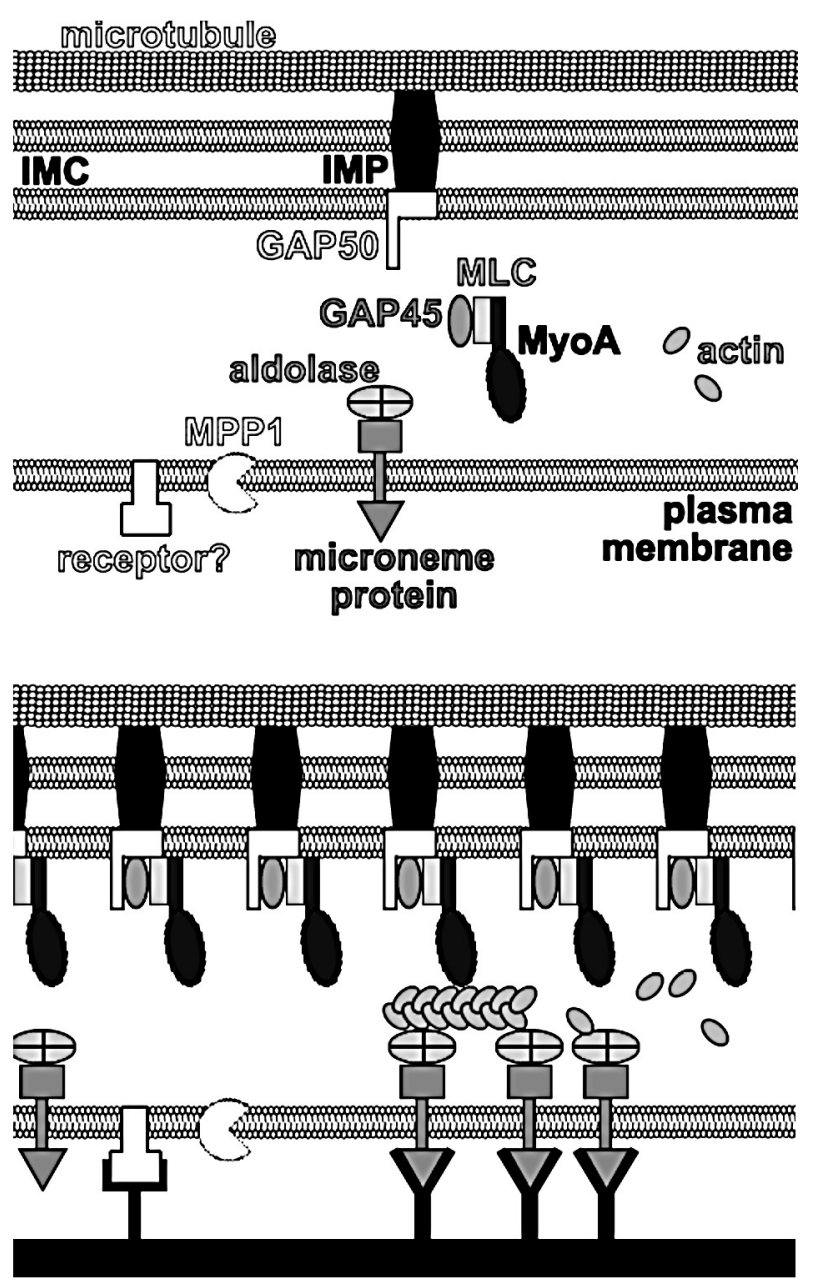

Fig. 1. - The glideosome comprises intracellular components as well as parts that are exposed to the extracellular milieu: the former includes the cytoskeleton, the actinomyosin complex and various interconnecting molecules that are highly conserved throughout the apicomplexans, whereas a large range of more divergent micronemal proteins and ligands with adhesive properties fall into the latter category.
Despite some major progress a number of unresolved issues still persist: 1. Actin dynamics: how is F-actin dynamics regulated? 2. Myosin motors: how many myosin motors contribute to the three forms of Toxoplasma motility and conoid protrusion during invasion? 3. Adhesins: what are the molecular bases for the broad range of host cell recognition and attachment by $T$. gondit? 4. Proteases: what are the proteases implicated in microneme proteins shedding during invasion and what is the functional significance of this event?

\section{ACTIN DYNAMICS}

F -actin has been difficult to detect in T. gondii, however the susceptibility of the parasite to actinpolymerizing and -depolymerizing drugs as well as molecular genetic studies (Dobrowolski \& Sibley, 1996; Wetzel et al., 2003) confirm that actin nucleation and polymerization is critical for motility. $T$. gondii contains a single conventional actin, similar to Cryptosporidium spp., while P. falciparum contains two distinct actin isoforms. Phylogenetic analysis reveals that apicomplexan actins differ substantially in residues that are important for monomer-monomer binding and biochemical studies indicate that these differences result in highly unusual kinetic properties (Sahoo et al., 2006; Schmitz et al., 2005; Schuler et al., 2005). T. gondii actin appears to be specially suited for rapid polymerization, yet it forms short unstable filaments, properties that are evidently adapted to its unique form of gliding. Consistent with these differences, apicomplexans contain relatively few conventional actin-binding proteins. Among their limited repertoire are actin depolymerizing factor (ADF) capping protein alpha and beta, profilin, Toxofilin (cofilin) and coronin. Unexpectedly, the apicomplexans lack a canonical Arp2/3 complex, which is otherwise widespread among eukaryotes to orchestrate actin assembly by nucleating filaments from the pointed end (Gordon \& Sibley, 2005; Schuler \& Matuschewski, 2006; Soldati \& Meissner, 2004). Apicomplexans but possess instead a number of actin-like and actin-related proteins, only a few of which are similar to known Arps (Gordon \& Sibley, 2005). Apicomplexans contain Arp1 homologues and all of the subunits of the dynactin complex, which links to the cytoplasmic motor, dynein and may function in apical transport of secretory vesicles, which are highly enriched at the apical end of the parasite. Apicomplexans also contain Arp4, and Arp6 homologues, which are likely involved in chromatin remodelling (Gordon \& Sibley, 2005). The remaining divergent actin like proteins may govern function unique to apicomplexans. Formin and profilin have recently been shown to provide an alternative mechanism for assembling F-actin (Higgs \& Peterson, 2005). T. gondii contains a profilin 
and three putative large formins that feature a typical FH2 and an only weakly conserved FH1 domain. Orthologues of the two putative formins TgFRM1 and TgFRM2 are evident in Plasmodium (Higgs \& Peterson, 2005) whereas TgFRM3 may represent a divergent, duplicated form of TgFRM2 (Daher et al., unpublished results). One of these orthologs, TgFRM1 is present in the conoid-enriched proteome (Hu et al., 2006) suggesting it may participate in apical actin polymerization. With only few exceptions, the same panoply of actin related and actin binding proteins are present in Plasmodium and other apicomplexan genomes (Baum et al., 2006a).

Genetics studies have demonstrated gliding motility as well host cell penetration and egress by $T$. gondii necessitate intact parasite actin filaments (Dobrowolski \& Sibley, 1996). In addition to cytochalasin D, which interferes with actin polymerization, jasplakinolide, a drug stabilizing actin filaments also has profound effects on motility (Wetzel et al., 2003) yet the mechanism by which apicomplexans control actin polymerization is unknown.

We have hypothesized that a formin/profilin (FRM/ PRF) system might orchestrate actin polymerization to promote gliding motility and assist parasite in invasion. The conditional disruption of $T$. gondii profilin gene $(\mathrm{TgPRF})$ has established that while TgPRF is not required for intracellular growth, the protein is indispensable for gliding motility, host cell invasion, active egress from host cells and virulence in mice (Plattner et al., 2008). Moreover, biochemical characterization of PRFs from Toxoplasma, Plasmodium and Cryptosporidium showed that these proteins function as bonafide profilin with regard to behaviour in actin polymerization assays despite the lack of affinity for poly-L-proline stretches. We conclude that TgPRF is likely to play a key role in regulating actin dynamics during invasion. All three TgFRM genes are expressed in tachyzoites and possess a FH2 domain while a putative FH1 (proline rich domain) is not readily recognizable. It is also of interest to note that the parasite formins lack the regulatory DAD domain but contain instead a tetratricopeptide domain suggesting possibly different mechanisms governing the regulation of the formin's function. Interestingly, both TgFRM1 and TgFRM2 are located in a chromosomal region mapped by the group of Dr Sibley as virulence locus conferring enhanced gliding and increased ability to migrate across biological barriers (Taylor et al., 2006). Antibodies raised against TgFRM1 and TgFRM2 have been used to demonstrate the dramatic relocalization of both proteins during invasion. Several fragments of TgFRM1 encompassing the FH2 domain have been expressed in bacteria and shown to exhibit a very potent activity in actin nucleation (Daher et al., unpublished). A recent study reported FRM1 localization and activity in P. falciparum and T. gondii (Baum et al., 2008).

\section{MYOSIN MOTORS}

T n T. gondii, 22 subpellicular microtubules emanates back from the microtubular organizing center called the conoid and confer a semi-rigid, spiral shape to the cell (Morrissette \& Sibley, 2002). The subpellicular microtubules are covered by an elaborate matrix of articulin-like proteins that are also found in ciliates. Together with flattened membranous cisternae, these articulin-like filaments comprise the inner membrane complex (IMC), which lies beneath the outer plasma membrane. The rigid cytoskeleton formed by these elements is likely important for maintaining cell shape during parasite motility and invasion, and for morphogenesis during cell division. A myosin motor of the class XIV, TgMyoA is essential to power movement and to propel the parasite into host cells (Meissner et al., 2002b). Two gliding associated proteins, TgGAP45 and TgGAP50, have been shown to anchor TgMyoA and its associated light chain TgMLC1 into the inner membrane complex (IMC) (Fig. 1) (Gaskins et al., 2004). Importantly, for gliding motility to occur, the current model proposes that the TgMyoA motor is firmly anchored to the subpellicular microtubule cytoskeleton of the parasite (Johnson et al., 2007), while actin is interacting via aldolase to the tails of microneme proteins, themselves solidly attached to substrate outside the cell via adhesive proteins (Fig. 1).

Our recent phylogenetic analysis of the repertoire of myosins in Apicomplexa and in others protozoans led us to postulate that other motors could potentially contribute to gliding motility (Foth et al., 2006) given the fact that $T$. gondii tachyzoites exhibit three distinct modes of motility (Hakansson et al., 1999). The T. gondii possesses the largest repertoire for each of the three types of motors found in any apicomplexan to date: 10 genes giving rise to at least 11 myosin heavy chains and 15 and 10 genes encode kinesin and dynein heavy chains, respectively. Phylogenetic analysis of the conserved head domains groups six $T$. gondii myosins into the alveolate-specific class XIV, two into the wellknown class VI, and one sequence each into the newly described myosin classes XXII, XXIII, and XXIV (Foth et al., 2006). We have showed that TgMyoD exhibits the same kinetics properties as TgMyoA but a conventional knockout has established that this motor does not play a significant role in motility of tachyzoites. However since this motor is primarily expressed in bradyzoites, it may fulfill an important role in this other invasive stage (Herm-Gotz et al., 2002). TgMyoB and TgMyoC are encoded by two alternatively spliced products of the same gene and are thought to be involved in cell division (Delbac et al., 2001; Gubbels et al., 2006). Other myosins contain protein domains such as a WD40 repeats and ATS1/RCC1-like domains in their 
tail region that had not been observed before as part of myosin motors (Foth et al., 2006). The biological function of the TgMHCs will await further investigations.

Myosin light chains (MLC) are binding to IQ domains usually positioned in the neck domain of myosin heavy chains (MHC). Although the contribution of the regulatory light chain to myosin II function is becoming clear, much less is known about the role played by MLC(s) in the regulation of unconventional myosins function. MLCs are thought to play a role in myosin regulation and/or in the stabilization of the neck domain, which is needed to produce steps of a definite size. While TgMLC1 interacts with TgMyoA (HermGotz et al., 2002), four putative additional light chains TgMLC2-5 are expressed in T. gondii (Frenal et al., unpublished). We have assembled these genes and determined the subcellular localisations of their products. TgMLC2, like TgMLC1 localizes to the IMC. Interestingly, experimental analyses of TgMLC1 and TgMLC2 revealed that their N-terminal domain serve as determinant for localization to the IMC and thus replaces classical role of MHC tail in bringing the motor to its site of action. Co-immunoprecipitation experiments have established that TgMLC2 belongs to a novel motor complex distinct from TgMoA-MLC1-GAP45-GAP50 and involving novel MHC and GAPs (Frenal et al., unpublished).

\section{ADHESINS}

T The apical complex is a characteristic of the invasive stages of all apicomplexan parasites and is the site of the successive regulated exocytosis of rhoptries and micronemes, as part of the invasion process (Carruthers \& Sibley, 1997). Micronemal proteins are apparently used for host-cell recognition, binding, and motility, while contents of the rhoptries appear to participate in the parasitophorous vacuole formation (Boothroyd \& Dubremetz, 2008; Dubremetz, 2007; Hakansson et al., 2001). The discharge by the micronemes is stimulated by a raise in intracellular Ca2+ (Carruthers et al., 1999; Lovett \& Sibley, 2003) but the natural triggering factor(s) and the signalling cascade involved are still unknown. To accommodate the broad host range specificity of $T$. gondii, adhesion might involve the recognition of ubiquitous surfaceexposed host molecules or, alternatively, the presence of various parasite attachment molecules able to recognize different host cell receptors.

Study of the adhesions and conventional or conditional knockouts of several microneme proteins (MICs) characterized so far indicate that these complexes are fulfilling non-overlapping functions. The MICs are pro- duced as multi-protein complexes in the endoplasmic reticulum and the transmembrane proteins serve as escorters, which contain sorting signals (Di Cristina et al., 2000) and thus assure the proper targeting of the complex to the micronemes. Three complexes have been described to date in T. gondii. TgMIC1/MIC4/MIC6 (Reiss et al., 2001) TgMIC3/MIC8 (Meissner et al., 2002a) and TgMIC2/M2AP (Rabenau et al., 2001) (Fig. 2A). TgMIC2 binds heparin in a MIDAS-independent manner and forms a hexamer complex (Harper et al., 2004). This complex is critical for gliding, and host cell attachment and invasion (Huynh \& Carruthers, 2006; Huynh et al., 2004). Parasites lacking TgMIC1 are severely impaired in their ability to attach to host cells and there is some synergism between TgMIC6/MIC2/MIC4 and TgMIC8/TgMIC3 complexes for virulence in vivo (Cerede et al., 2005). Like mic1ko, parasite lacking TgMIC4 or TgMIC6, are impaired in host cell attachment and invasion (Friedrich et al., unpublished). The disruption of TgMIC8 reveals that this mutant is dramatically impaired in invasion but apparently not in gliding or host cell attachment. Interestingly, this mutant is unable to form the moving junction and does not release the rhoptry proteins (Kessler et al., 2008).

Finally, TgAMA1 appears to play a different but critical role in the formation of the moving junction at the point of contact between the parasite and the host cell membranes during invasion (Mital et al., 2005). In addition, TgAMA1 has recently been shown to co-operates with rhoptry neck proteins in the formation and maintenance of the moving junction during host cell invasion (Fig. 2A) (Alexander et al., 2005; Lebrun et al., 2005).

We have recently dissected structurally and functionally the TgMIC4-1-6 complex and discovered interesting findings. TgMIC1 is a remarkable, multifunctional protein that not only interacts simultaneously with TgMIC4, TgMIC6 and host cells but is also essential for proper folding and transport of the whole complex through the early secretory pathways. Another unusual feature of TgMIC1 is that, unlike the battery of other micronemal proteins, its sequence does not exhibit a clear likeness to vertebrate adhesive motifs. The NMR structure revealed a previously unidentified and novel Galectin domain within the C-terminus of TgMIC1 that recruits correctly folded TgMIC6 to the complex (Saouros et al., 2005a; Saouros et al., 2005b). Further structural analysis of the N-terminal domain of TgMIC1 releaved again a novel fold (MAR). The screening of carbohydrates chips with this domain identified sialic acid as the binding entity. The binding of MAR to sialic acid on host cells was confirmed by performing host cell-binding assays in the presence of increasing concentration of sialic acid and by pre-treating the host cells with neuraminidase. These results confirmed the previously reported importance of host cell surface sialic acid resi- 
dues on the process of $T$. gondii invasion (Monteiro et al., 1998). We observed that MAR domains exist in tandem repeats, which provide a highly specialized structure for glycan discrimination. Our work uncovers new features of parasite receptor interactions at the early stages of host cell invasion (Blumenschein et al., 2007). Sialic acid exposing receptors are commonly used by pathogens (including viruses, Trypanosomes and Plasmodium species) as major determinant for invasion. The $P$. falciparum erythrocyte-binding antigen 175 (PfEBA175) is one of the best-characterized merozoite ligand, which binds to the terminal sialic acids on the erythrocyte glycoprotein glycophorin A (Chattopadhyay et al., 2006).
TgMIC2/M2AP

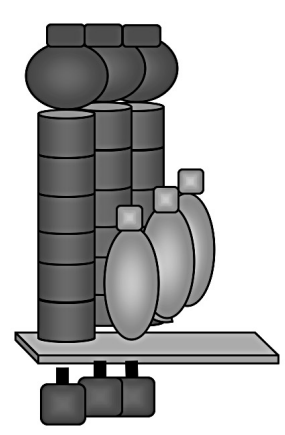

heparin/MIC2
TgMIC6/MIC1/MIC4

A

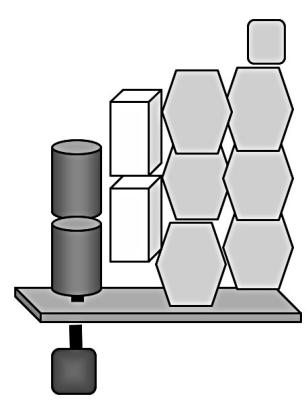

Receptor/ligand

\section{PROTEASES}

R emarkably, most MICs are proteolytically cleaved during their biogenesis and/or post-exocytosis Cleavage occuring during their biogenesis and transport to the micronemes and the significance of the processing occurring along the secretory pathway is linked to the specificity of complex formation, biogenesis, targeting to the organelle and masking of enzymatically active sites. After discharge by the organelle, further cleavages cause the dissolution of the microneme protein complexes and their release from the parasite surface (Fig. 2B). The post-exocytosis proces-
TgMIC8/TgMIC3

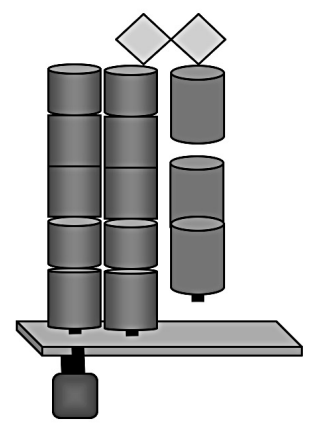

TgAMA-/TgRON2/TgRON4 sialic acid/MIC1
Lectin/MIC3/8

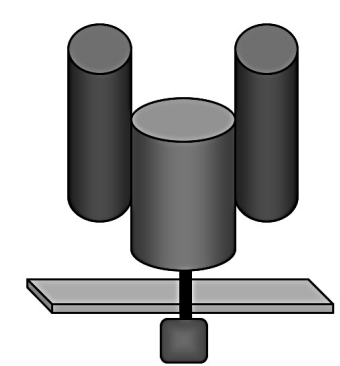

TgMIC2/M2AP
TgMIC6/MIC1/MIC4

B

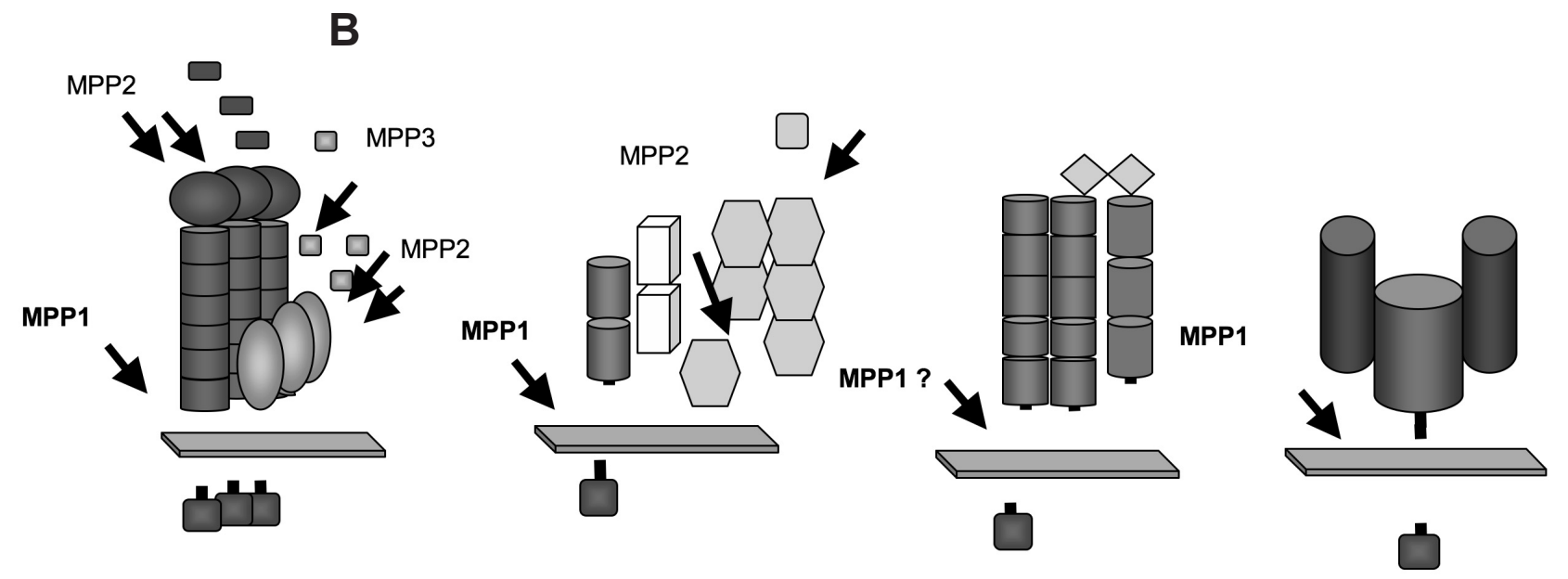

Fig. 2. - A. The major T. gondii protein complexes released on parasite surface during invasion. B. Proteolytic processing of MIC post exocytosis. 
sing events are likely to be directly linked to the host cell attachment and invasion process (Dowse \& Soldati, 2004). Studies into the processing of the type I transmembrane protein TgMIC2 revealed that, following its release from the micronemes and redistribution across the plasma membrane, the protein is shed from the parasite surface. This shedding was found to result from a proteolytic cleavage near the C-terminus by the action of a protease named Microneme Protein Protease 1 (MPP1) (Carruthers et al., 2000; Carruthers \& Sibley, 1999). Inhibition of MPP1 cleavage provided indirect evidence that this activity is essential for invasion in T. gondii (Brossier et al., 2003). The first development towards discovering the identity of MPP1 was the identification of intramembrane proteolytic cleavage site (IA $\downarrow$ GG) on TgMIC6 targeted by the protease (Opitz et al., 2002). It was observed that other transmembrane MICs are also cleaved near their C-terminus, resulting in their shedding (Howell et al., 2005; Zhou et al., 2004), and this led to the obvious suspicion that MPP1 is responsible for shedding several, if not all transmembrane TgMICs. An examination of the list of intramembrane protease families so far discovered immediately suggested the rhomboid-like family as candidate enzymes for MPP1. Rhomboid-1 from Drosophila, the founding member of the family is an intramembrane-cleaving protease (ICliP), which regulates the secretion of an EGF-receptor ligand, Spitz, via cleavage in the Spitz TM domain. The cleavage of TgMICs by the Drosophila enzyme provided the evidence in support of MPP1 being a rhomboid-like protease (Urban \& Freeman, 2003). T. gondii possesses six rhomboid-like proteins that belong to the family of intramembrane-cleaving serine proteases and TgROM 4 sand TgROM5 constitute the two primary candidates for MPP1 activity (Brossier et al., 2005; Dowse et al., 2005). Indeed MPP1 was anticipated to be constitutively active at the parasite surface. Both studies performed analyses of their localization. TgROM1 localises to the micronemes, TgROM2 to the Golgi, and TgROM4 to the parasite surface. TgROM3 does not appear to be expressed in tachyzoites, the life-cycle stage where MPP1 was described, and was therefore excluded from further analysis. TgROM6 is a mitochondrial protein as predicted from a phylogenetic analysis and experimentally confirmed (Sheiner et al., unpublished). Brossier et al., observed that TgROM5 is on the parasite surface and concentrated towards the posterior end of the extracellular parasite. This, combined with data, which showed cleavage of TgMIC2 by TgROM5 in a heterologous cell-based assay, led the authors to suggest that TgROM5 is the primary candidate for MPP1. The importance and exact contribution of the intramembrane cleavage in the invasion process could be best elucidated by the generation of a conditional knockout in $T$. gondii where it is possible to determine the precise step of the process, which is blocked (gliding, penetration, termination of invasion and egress). Currently, there is still a degree of uncertainty about the identity of MPP1 in T. gondii (TgROM4 or TgROM5) however the situation is more clear in Plasmodium, which does not possess a ROM5 equivalent. In the malaria parasites, PfAMA-1 and PfMSP1 are shedded by the subtilase PfSUB2. PfROM4 is most likely responsible for the cleavage of the EBA175 family members that are responsible for the alternative invasion pathways in red blood cells (Baker et al., 2006; O'Donnell et al., 2006). Since PfROM4 is presumably cleaving all members of the EBA family responsible for the alternative invasion pathways of the red blood cell, this unique gene might represent the Achilles heel of the malaria parasites. The aim here is to validate ROM 4 as potential target for drug or vaccine development. In T. gondii, most of the membrane spanning MICs appear to be cleaved in the membrane and it is plausible that some could be cleaved by two distinct proteases justifying the existence of TgROM 4 and TgROM5.

\section{CONCLUDING REMARKS}

nvasion factors such as regulators of actin dynamics, myosins motors, microneme proteins and proteases are conserved and exhibit features that are restricted to this important group of pathogens. Understanding their mode of action at the molecular level shall not only shed light on basic cellular processes such as cell motility and cell-cell interactions but might also reveal new targets for therapeutic interventions. The demonstration of the essential character of a gene is a prerequisite for its validation as target for the development of new drugs or vaccines. Furthermore, a deep understanding of the structure and function of such a gene product is most suitable to accelerate the phase of improved efficacy of the intervention.

\section{ACKNOWLEDGEMENTS}

$\mathrm{F}$ $\mathrm{B}$ is supported by the Swiss National Foundation FN 3100A0 - 102255/DS and currently 3100A0$116722 /$ DS. It is part of the activities of the BioMalPar European Network of Excellence supported by a European grant (LSHP-CT-2004-503578) from the Priority 1 "Life Sciences, Genomics and Biotechnology for Health" in the $6^{\text {th }}$ Framework Programme and DS is an international scholar of the Howard Hughes Medical Institutes.

\section{REFERENCES}

Alexander D.L., Mital J., Ward G.E., Bradley P. \& Boothroyd J.C. Identification of the moving junction complex of 
Toxoplasma gondii: a collaboration between distinct secretory organelles. PLoS Pathog, 2005, 1 (2), e17.

Baker R.P., Wijetilaka R. \& Urban S. Two Plasmodium rhomboid proteases preferentially cleave different adhesins implicated in all invasive stages of malaria. PLoS Pathog., 2006, 2 (10), e113.

Baum J., Papenfuss A.T., Baum B., Speed T.P. \& Cowman A.F. Regulation of apicomplexan actin-based motility. Nat. Rev. Microbiol., 2006a, 4 (8), 621-628.

Baum J., Richard D., Healer J., Rug M., Krnajski Z., Gilberger T.W., Green J.L., Holder A.A. \& Cowman A.F. A conserved molecular motor drives cell invasion and gliding motility across malaria life cycle stages and other apicomplexan parasites. J. Biol. Chem., 2006b, 281 (8), 5197-5208.

Baum J., Tonkin C.J., Paul A.S., Rug M., Smith B.J., Gould S.B., Richard D., Pollard T.D. \& Cowman A.F. A malaria parasite formin regulates actin polymerization and localizes to the parasite-erythrocyte moving junction during invasion. Cell. Host. Microbe, 2008, 3 (3), 188-198.

Blumenschein T.M., Friedrich N., Childs R.A., SAOUros S., CarPenter E.P., Campanero-Rhodes M.A., Simpson P., ChaI W., Koutroukides T., Blackman M.J., Feizi T., Soldati-Favre D. \& MatThews S. Atomic resolution insight into host cell recognition by Toxoplasma gondii. Embo J., 2007, 26 (11), 28082820 .

Boothroyd J.C. \& Dubremetz J.F. Kiss and spit: the dual roles of Toxoplasma rhoptries. Nat. Rev. Microbiol., 2008, 6 (1), 79-88.

Bradley P.J. \& Sibley L.D. Rhoptries: an arsenal of secreted virulence factors. Curr. Opin. Microbiol., 2007, 10 (6), 582587.

Brossier F., Jewett T.J., Lovett J.L. \& Sibley L.D. C-terminal processing of the toxoplasma protein MIC2 is essential for invasion into host cells. J. Biol. Chem., 2003, 278 (8), 62296234 .

Brossier F., Jewett T.J., Sibley L.D. \& Urban S. A spatially localized rhomboid protease cleaves cell surface adhesins essential for invasion by Toxoplasma. Proc. Natl. Acad. Sci. USA, 2005, 102 (11), 4146-4151.

Buscaglia C.A., Coppens I., Hol W.G. \& Nussenzweig V. Sites of interaction between aldolase and thrombospondin-related anonymous protein in Plasmodium. Mol. Biol. Cell., 2003, 14 (12), 4947-4957.

Buscaglia C.A., Penesetti D., Tao M. \& Nussenzweig V. Characterization of an aldolase-binding site in the WiskottAldrich syndrome protein. J. Biol. Chem., 2006, 281 (3), 1324-1331.

Carruthers V. \& Boothroyd J.C. Pulling together: an integrated model of Toxoplasma cell invasion. Curr. Opin. Microbiol., 2007, 10 (1), 83-89.

Carruthers V.B., Moreno S.N. \& Sibley L.D. Ethanol and acetaldehyde elevate intracellular $\left[\mathrm{Ca}_{2}^{+}\right]$and stimulate microneme discharge in Toxoplasma gondii. Biochem. J., 1999, 342 (Pt 2), 379-386.

Carruthers V.B., Sherman G.D. \& Sibley L.D. The Toxoplasma adhesive protein MIC2 is proteolytically processed at multiple sites by two parasite-derived proteases. J. Biol. Chem., 2000, 275 (19), 14346-14353.
Carruthers V.B. \& Sibley L.D. Sequential protein secretion from three distinct organelles of Toxoplasma gondii accompanies invasion of human fibroblasts. Eur. J. Cell. Biol., 1997, 73 (2), 114-123.

CarRuthers V.B. \& Sibley L.D. Mobilization of intracellular calcium stimulates microneme discharge in Toxoplasma gondii. Mol. Microbiol., 1999, 31 (2), 421-428.

Cerede O., Dubremetz J.F., Soete M., Deslee D., Vial H., Bout D. \& Lebrun M. Synergistic role of micronemal proteins in Toxoplasma gondii virulence. J. Exp. Med., 2005, 201 (3), 453-463.

Chattopadhyay D., Rayner J., McHenry A.M. \& Adams J.H. The structure of the Plasmodium falciparum EBA175 ligand domain and the molecular basis of host specificity. Trends Parasitol, 2006, 22 (4), 143-145.

Delbac F., Sanger A., Neuhaus E.M., Stratmann R., Ajioka J.W., Toursel C., Herm-Gotz A., Tomavo S., Soldati T. \& SolDATI D. Toxoplasma gondii myosins B/C: one gene, two tails, two localizations, and a role in parasite division. J. Cell. Biol., 2001, 155 (4), 613-623.

Di Cristina M., Spaccapelo R., Soldati D., Bistoni F. \& CriSANTI A. Two conserved amino acid motifs mediate protein targeting to the micronemes of the apicomplexan parasite Toxoplasma gondii. Mol. Cell. Biol., 2000, 20 (19), 7332-7341.

DOBROWOLSKI J.M. \& SIBLEY L.D. Toxoplasma invasion of mammalian cells is powered by the actin cytoskeleton of the parasite. Cell, 1996, 84 (6), 933-939.

Dowse T. \& SOldati D. Host cell invasion by the apicomplexans: the significance of microneme protein proteolysis. Curr. Opin. Microbiol., 2004, 7 (4), 388-396.

Dowse T.J., Pascall J.C., Brown K.D. \& Soldati D. Apicomplexan rhomboids have a potential role in microneme protein cleavage during host cell invasion. Int. J. Parasitol., 2005, 35 (7), 747-756.

DubREMETZ J.F. Rhoptries are major players in Toxoplasma gondii invasion and host cell interaction. Cell. Microbiol., 2007, 9 (4), 841-848.

Foth B.J., Goedecke M.C. \& Soldati D. New insights into myosin evolution and classification. Proc. Natl. Acad. Sci. USA, 2006, 103 (10), 3681-3686.

Gaskins E., Gilk S., DeVore N., Mann T., Ward G. \& Beckers C. Identification of the membrane receptor of a class XIV myosin in Toxoplasma gondii. J. Cell. Biol., 2004, 165 (3), 383-393.

Gordon J.L. \& Sibley L.D. Comparative genome analysis reveals a conserved family of actin-like proteins in apicomplexan parasites. BMC Genomics, 2005, 6, 179.

Gubbels M.J., Vaishnava S., Boot N., Dubremetz J.F. \& StriePEN B. A MORN-repeat protein is a dynamic component of the Toxoplasma gondii cell division apparatus. J. Cell. Sci., 2006, 119 (Pt 11), 2236-2245.

Hakansson S., Charron A.J. \& Sibley L.D. Toxoplasma evacuoles: a two-step process of secretion and fusion forms the parasitophorous vacuole. Embo J., 2001, 20 (12), 31323144 .

Hakansson S., Morisaki H., Heuser J. \& Sibley L.D. Time-lapse video microscopy of gliding motility in Toxoplasma gondii 
reveals a novel, biphasic mechanism of cell locomotion. Mol. Biol. Cell., 1999, 10 (11), 3539-3547.

Harper J.M., Zhou X.W., Pszenny V., Kafsack B.F. \& CarruTHERS V.B. The novel coccidian micronemal protein MIC11 undergoes proteolytic maturation by sequential cleavage to remove an internal propeptide. Int. J. Parasitol., 2004, 34 (9), 1047-1058.

Herm-Gotz A., Weiss S., Stratmann R., Fujita-Becker S., Ruff C., Meyhofer E., Soldati T., Manstein D.J., GeEves M.A. \& SolDATI D. Toxoplasma gondii myosin A and its light chain: a fast, single-headed, plus-end-directed motor. Embo J., 2002, 21 (9), 2149-2158.

Higgs H. \& Peterson K.J. Phylogenetic anlayis of the formin homology 2 domain. Mol. Biol. Cell., 2005, 16, 1-13.

Hoff E.F. \& CARruthers V.B. Is Toxoplasma egress the first step in invasion? Trends Parasitol., 2002, 18 (6), 251-255.

Howell S.A., Hackett F., Jongco A.M., Withers-Martinez C., Kim K., Carruthers V.B. \& Blackman M.J. Distinct mechanisms govern proteolytic shedding of a key invasion protein in apicomplexan pathogens. Mol. Microbiol., 2005, 57 (5), 1342-1356.

Hu K., Johnson J., Florens L., Fraunholz M., Suravajjala S., Dilullo C., Yates J., Roos D.S. \& Murray J.M. Cytoskeletal components of an invasion machine - The apical complex of Toxoplasma gondii. PLoS Pathog., 2006, 2 (2), e13.

HuYnh M.H. \& CARRUTHERS V.B. Toxoplasma MIC2 is a major determinant of invasion and virulence. PLoS Pathog., 2006, 2 (8).

Huynh M.H., Opitz C., Kwok L.Y., Tomley F.M., Carruthers V.B. \& SOLDATI D. Trans-genera reconstitution and complementation of an adhesion complex in Toxoplasma gondii. Cell. Microbiol., 2004, 6 (8), 771-782.

JewETT T.J. \& SibLEy L.D. Aldolase forms a bridge between cell surface adhesins and the actin cytoskeleton in apicomplexan parasites. Mol. Cell., 2003, 11 (4), 885-894.

Johnson T.M., Rajfur Z., Jacobson K. \& Beckers C.J. Immobilization of the type XIV myosin complex in Toxoplasma gondii. Mol. Biol. Cell., 2007, 18 (8), 3039-3046.

Joiner K.A., Fuhrman S.A., MietTinen H.M., Kasper L.H. \& Mellman I. Toxoplasma gondii: fusion competence of parasitophorous vacuoles in Fc receptor-transfected fibroblasts. Science, 1990, 249 (4969), 641-646.

KeEley A. \& Soldati D. The glideosome: a molecular machine powering motility and host-cell invasion by Apicomplexa. Trends Cell. Biol., 2004, 14 (10), 528-532.

Kessler H., Herm-Götz A., Hegge S., Rauch M., Soldati D., FRISCHKNECHT F. \& MEISSNER I. A protein required for sequential discharge of secretory organelles during host cell invasion by Toxoplasma gondii. J. Cell. Sci., 2008, 121, 947956.

Lebrun M., Michelin A., El Hajj H., Poncet J., Bradley P.J., Vial H. \& Dubremetz J.F. The rhoptry neck protein RON4 re-localizes at the moving junction during Toxoplasma gondii invasion. Cell. Microbiol., 2005, 7 (12), 1823-1833.

LovetT J.L. \& Sibley L.D. Intracellular calcium stores in Toxoplasma gondii govern invasion of host cells. J. Cell. Sci., 2003, 116 (Pt 14), 3009-3016.
Meissner M., Reiss M., Viebig N., Carruthers V.B., Toursel C., Tomavo S., Ajioka J.W. \& Soldati D. A family of transmembrane microneme proteins of Toxoplasma gondii contain EGF-like domains and function as escorters. J. Cell. Sci., 2002, 115, 563-574.

Meissner M., Schluter D. \& Soldati D. Role of Toxoplasma gondii myosin A in powering parasite gliding and host cell invasion. Science, 2002, 298 (5594), 837-840.

Mital J., Meissner M., Soldati D. \& Ward G.E. Conditional expression of Toxoplasma gondii apical membrane antigen-1 (TgAMA1) demonstrates that TgAMA1 plays a critical role in host cell invasion. Mol. Biol. Cell., 2005, 16 (9), 4341-4349.

Monteiro V.G., Soares C.P. \& De Souza W. Host cell surface sialic acid residues are involved on the process of penetration of Toxoplasma gondii into mammalian cells. FEMS Microbiol. Lett., 1998, 164 (2), 323-327.

Morrissette N.S. \& Sibley L.D. Cytoskeleton of apicomplexan parasites. Microbiol. Mol. Biol. Rev., 2002, 66 (1), 21-38.

O’Donnell R.A., Hackett F., Howell S.A., Treeck M., Struck N., Krnajski Z., Withers-Martinez C., Gilberger T.W. \& BlackMAN M.J. Intramembrane proteolysis mediates shedding of a key adhesin during erythrocyte invasion by the malaria parasite. J. Cell. Biol., 2006, 174 (7), 1023-1033.

Opitz C., Di Cristina M., Reiss M., Ruppert T., Crisanti A. \& SOLDATI D. Intramembrane cleavage of microneme proteins at the surface of the apicomplexan parasite Toxoplasma gondii. Embo J., 2002, 21 (7), 1577-1585.

Plattner F., Yarovinsky F., Romero S., Didry D., Carlier M.F., SHER A. \& SOldati-Favre D. Toxoplasma profilin is essential for host cell invasion and TLR11-dependent induction of an interleukin-12 response. Cell Host \& Microbe, 2008, $3,77-87$

Rabenau K.E., Sohrabi A., Tripathy A; Reitter A., Ajioka J.W., TOMley F.M. \& Carruthers V.B. TgM2AP participates in Toxoplasma gondii invasion of host cells and is tightly associated with the adhesive protein TgMIC2. Mol. Microbiol., 2001, 41, 1-12.

Reiss M., Viebig N., Brecht S., Fourmaux M.N., Soete M., Di Cristina M., Dubremetz J.F. \& Soldati D. Identification and characterization of an escorter for two secretory adhesins in Toxoplasma gondii. J. Cell. Biol., 2001, 152 (3), 563-578.

Roos D.S., Donald R.G., Morrissette N.S. \& Moulton A.L. Molecular tools for genetic dissection of the protozoan parasite Toxoplasma gondii. Methods Cell. Biol., 1994, 45, $27-63$.

Sahoo N., Beatty W., Heuser J., Sept D. \& Sibley L.D. Unusual kinetic and structural properties control rapid assembly and turnover of actin in the parasite Toxoplasma gondii. Mol. Biol. Cell., 2006, 17 (2), 895-906.

Saouros S., Chen H.A., Simpson P., Cota E., Edwards-Jones B., SOldati-Favre D. \& MatThews S. Complete resonance assignments of the C-terminal domain from MIC1: a micronemal protein from Toxoplasma gondii. J. Biomol. NMR, 2005a, 31 (2), 177-178.

Saouros S., Edwards-Jones B., Reiss M., Sawmynaden K., Cota E., Simpson P., Dowse T.J., Jakle U., Ramboarina S., Shivarattan T., MatThEws S. \& Soldati-Favre D. A novel galectin-like domain 
from Toxoplasma gondii micronemal protein 1 assists the folding, assembly, and transport of a cell adhesion complex. J. Biol. Chem., 2005b, 280 (46), 38583-38591.

Schmitz S., Grainger M., Howell S., Calder L.J., Gaeb M., Pinder J.C., Holder A.A. \& Veigel C. Malaria parasite actin filaments are very short. J. Mol. Biol., 2005, 349 (1), 113125.

SChUlER H. \& MATUSCHEWski K. Regulation of apicomplexan microfilament dynamics by a minimal set of actin-binding proteins. Traffic, 2006, 7 (11), 1433-1439.

Schuler H., Mueller A.K. \& Matuschewski K. Unusual properties of Plasmodium falciparum actin: new insights into microfilament dynamics of apicomplexan parasites. FEBS Lett., 2005, 579 (3), 655-660.

Sibley L.D. Intracellular parasite invasion strategies. Science, 2004, 304 (5668), 248-253.

Soldati D., Foth B.J. \& Cowman A.F. Molecular and functional aspects of parasite invasion. Trends Parasitol., 2004, 20 (12), 567-574.

Soldati D. \& MeIssner M. Toxoplasma as a novel system for motility. Curr. Opin. Cell. Biol., 2004, 16 (1), 32-40

Taylor S., Barragan A., Su C., Fux B., Fentress S.J., Tang K., Beatty W.L., HajJ H.E., Jerome M., Behnke M.S., White M., Wooton J.C. \& Sibley L.D. A secreted serine-threonine kinase determines virulence in the eukaryotic pathogen Toxoplasma gondii. Science, 2006, 314 (5806), 1776-1780.

URBAN S. \& FREEMAN M. Substrate specificity of rhomboid intramembrane proteases is governed by helix-breaking residues in the substrate transmembrane domain. Mol. Cell., 2003, 11 (6), 1425-1434.

Wetzel D.M., HaKansson S., Hu K., Roos D. \& Sibley L.D. Actin filament polymerization regulates gliding motility by apicomplexan parasites. Mol. Biol. Cell., 2003, 14 (2), 396406.

Zhou X.W., Blackman M.J., Howell S.A. \& Carruthers V.B. Proteomic analysis of cleavage events reveals a dynamic two-step mechanism for proteolysis of a key parasite adhesive complex. Mol. Cell. Proteomics, 2004, 3 (6), 565576. 